\title{
Formation Mechanism Analysis and Detection of Charged Particles in an Aero-engine Gas Path
}

\author{
Zhenhua Wen*, Junxing Hou** and ZhiQiang Jiang*** \\ School of Mechatronics Engineering, Zhengzhou Institute of Aeronautical Industry Management, Zhengzhou, China
}

\begin{abstract}
The components of an aero-engine gas path cannot be monitored in a timely way due to a lack of real-time monitoring technologies. As an attempt to address this problem, we have conducted research on a condition monitoring technology based on the charging characteristics of particles in an aero-engine gas path, and emphatically analyze the formation of particles in an aero-engine gas path, the charging mechanism of carbon particles and the factors that influence the charge quantity and polarity. The verification experiments are performed on the simulated experiment platform and a turbo-shaft engine test bench. The results show the carbon particles' carry charge, and an obvious change in the total electrostatic charge level in the aero-engine gas path due to the increased carbon particles produced by burning or abnormal metal particles; the charge number is related to the size of particles, and the bigger carbon particles carry a negative charge and metal particles carry a positive charge; the change in engine power can lead to an obvious change in the level of electrostatic charge in the gas path, and the change in electrostatic charge results from the extra carbon particles formed in the rich-oil burning process. The research provides a reference for establishing the baseline of electrostatic charge while the engine runs on different power. The study also demonstrates the validity of the electrostatic monitoring technology and establishes a base for developing the application of electrostatic monitoring technology in aero-engines.
\end{abstract}

Key words: Aero-Engine, Electrostatic Monitoring, Condition Monitoring, Charging Particles

\section{Acronym and Abbreviation}

PHM = Prognostics and health management

NI = National Instruments

$$
\begin{array}{ll}
e & =\text { Electronic charge } \\
v & =\text { Particles' velocity } \\
N & =\text { Ionic concentration } \\
t & =\text { Time }
\end{array}
$$

\section{Introduction}

The key components of an aero-engine gas path operate under the rugged environment of the high temperature, high pressure and high rotation speed, and there are fewer effective real-time monitoring methods to detect their work status. Consequently, they have been the primary fault source $[1,2]$. The current research shows that the faults caused by the gas path component of an aero-engine account for more than 90 percent of all the faults, and the maintenance cost
This is an Open Access article distributed under the terms of the Creative Commons Attribution Non-Commercial License (http://creativecommons.org/licenses/by$\mathrm{nc} / 3.0 /$ which permits unrestricted non-commercial use, distribution, and reproduction in any medium, provided the original work is properly cited. cc * Ph.D., Corresponding Author: levinzhwen@126.com

** Ph D.

*** Professor 
for it almost accounts for more than 60 percent of the total maintenance cost of an engine. The real-time condition monitoring of gas path components must be achieved in order to realize prognostics and health management (PHM) for aero-engines. Aero-engine gas path electrostatic monitoring technology is a real-time online monitoring tool, which can provide early warning information by monitoring the changes of the total electrostatic level in the engine gas path. The technology was first presented in the literature by Robert Couch, and the research shows turbine engine gaspath failures can be predicted four or more hours ahead of time by the electrostatic monitoring technology [3], because the electrostatic monitoring technology detects changes in the charge level produced by the additional particles, such as the ingested debris and gas path component faults. Therefore, its advantage is that it measures the direct product of the fault rather than the secondary effects such as the increased vibration or temperature [4]. At present, the technology has been successfully applied in the F35 program. The technology has also been researched in recent years by some researchers in China [5]. They focus on the electrostatic monitoring principle [6], the sensor principle [7], and some signal processing methods [8]. A prototype of the electrostatic sensor for gas path monitoring was developed [9] and used in the engine test-bench [10].

\section{Source of the charged particles in the aero-engine gas path}

Generally, the exhaust emissions of aero-engines mainly include carbon dioxide, water vapor, nitrogen oxides, hydrocarbons, carbon monoxide, sulfur oxides, and carbon particles, as shown in Fig. 1.The particles are chiefly carbon particles generated by combustion. They are often mixed with some particles generated by the damage and the fault of the hot parts of aero-engines. The formation of particles and the charging mechanism are predominantly analyzed in the following section.

\subsection{Formation mechanisms of carbon particles}

The carbon particles are formed in the primary

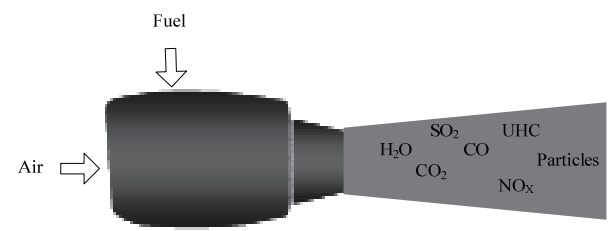

Fig. 1. Combustion products in the engine exhaust emissions combustion zone of the combustion chamber. They are then partially oxidized in the intermediate zone and the mixing zone. The generation process of carbon particles often experiences nucleation, surface growth and aggregation, and agglomeration, accompanied by the oxidation process.

In the oil-rich zone of the combustion chamber, the combustion of hydrocarbons can be described by the following:

$$
\mathrm{C}_{n} \mathrm{H}_{m}+k \mathrm{O}_{2} \rightarrow 2 k \mathrm{CO}+\frac{m}{2} \mathrm{H}_{2}+(n-2 k) \mathrm{C}
$$

where $C$ represents solid-state carbon. In this case, solid carbon appears under the condition of $n>2 k$ or $n_{c} / n_{o}>1$. The surface reaction between the core of the carbon particles and molecules in the gas phase contributes to the increase of the mass of particles, and the process is the particle surface growth. Moreover, particle aggregation is another way for growth. As the growth rate is very rapid, the typical time it takes to generate carbon is $10^{-4}$ second. After the formation of carbon particles, its oxidation or burnout rate is much slower than the generation. The reaction rate can be expressed by the following empirical formula:

$$
k^{\prime}=8.1 \times 10^{4} e^{-\frac{37000}{R T}}
$$

where $R$ is the gas constant, $T$ is the combustion temperature $\left({ }^{\circ} \mathrm{C}\right)$. Shown by the equation above, the carbon particles are formed in the condition where the temperature is 900 $\sim 1000^{\circ} \mathrm{C}$ and the air is insufficient. The air fuel is mixed poorly in the local zone despite sufficient air, and this will result in the generation of carbon particles. In addition, the generation of carbon particles is related to the properties of the fuel, combustion pressure, combustion temperature, gas-fuel ratio, the atomization and the spray pattern, so generally we think that carbon particle formation is inevitable [11].

\subsection{The charging mechanism of carbon particles}

The formation of the particles usually occurs in the combustion chamber, and due to the high temperature in the combustion process and its chemical ionization, thermal ionization is also completed in this region. There are severe and complex multi-step chemical reactions between the fuel and oxygen when an aero-engine runs, and the typical chemical reaction is as follows [11] :

$$
\begin{aligned}
& \mathrm{CH}+\mathrm{O}-\mathrm{CHO}^{+}+e^{-} \\
& \mathrm{CHO}+\mathrm{H}_{2} \mathrm{O}^{-} \mathrm{H}_{3} \mathrm{O}+\mathrm{CO}+e \\
& \mathrm{CH}+\mathrm{C}_{2} \mathrm{H}_{2}-\mathrm{C}_{3} \mathrm{H}_{3}+e^{-}
\end{aligned}
$$


$\mathrm{CH}_{3}{ }^{+}, \mathrm{CH}_{3} \mathrm{O}^{+}, \mathrm{C}_{3} \mathrm{H}_{3}{ }^{+}, \mathrm{H}_{3} \mathrm{O}^{+}, \mathrm{NO}^{+}$will form in the combustion process. Shown by the formulas (3) to (5), ions and a large number of electrons are generated synchronously in the reactions, and they are present in the flame zone and burnt zone. The concentration of ions and free electrons change constantly as the combustion process changes in the combustion chamber. In general, the carbon particles charge mainly through thermal ionization and electric adsorption; carbon particles can obtain a charge from the thermal emission of electrons, and can also interact with the ion produced by chemical ionization and the adsorb charge, and the charge may increase with the absorption of ions and the reaction with the various types of ions. The positive and negative ions in the aero-engine exhaust are measured in experimental studies [12-14], which also analyze the effect of the fuel flow and sulfur content on the quantity of the electrostatic charge. The experimental results show: (1) The concentration of negative ions increases with the increase of fuel sulfur content, and in the case of low flow in particular, the concentration of positive ions is affected less. (2) Charge characteristics of carbon particles and the relative ratio of the carbon particles in the combustor exit depend not only on the concentration of electrons and ions, but also on the size of the particles. (3) Large carbon particles carry the positive charge in the high-temperature region close to the flame due to thermal ionization. The number of particles with a positive charge is larger than that of a particle with a negative charge in the outlet of the combustion chamber. (4) The carbon particles interact with the electron after passing through the flame zone. The carbon particles mainly carry negative charge due to the predominant interaction between the small carbon particles (particle diameter of less than $3 \mathrm{~nm}$ ) and electronics. In other words, the small carbon particles are mainly negatively charged with electricity under the condition of the relatively stable and sufficient combustion. This can be seen from the above analysis that indicates that many ions are generated in the combustion process with chemical ionization, thermal ionization and other physical ionization. Particles absorb and interact with these free ions and then charge. The formation of carbon particles, charge mode and charge quantity are inseparable to the fuel combustion process in the combustion chamber. Therefore, the charge characteristics of carbon particles reflect the combustion performance of the engine to a certain extent. Next, the mechanism of abnormal particle matter generated in the gas path is analyzed.

\subsection{The charge mechanism of abnormal particles}

In addition to the combustion of carbon particles, other abnormal particles are present mainly due to the component faults in the aero-engine gas path, and the charging process of these abnormal particles follows the theory of the contact-separation charging mechanism. Contact charging is the process of forming the electric double layer, in which the difference of the work function determines the charging polarity of the two objects. The object with a small work function is positively charged, and the object with a large work function is negatively charged. The separation is to separate the contact object. The process is accompanied by an electrostatic capacitance decrease and potential rise. The friction is the process of continuous contacts and separates in the different contact points of the contact surfaces between two objects. Therefore, the triboelectric charging is also one of the contactseparation charging mechanisms. However, the process of the triboelectric charging is much more complex than single contact-separation charging, because the friction can cause the temperature to rise, and mechanical fracture and thermal decomposition of the molecules change the charge quantity of the object. In addition, friction velocity, friction time, friction area, and pressure affect the charge quantity of the object. Thus, the charging quantity caused by contact charging depends on three factors: the contact process, the separation process and the friction effect. As for the gas path components of an aero-engine, such as the blades, when friction fault occurs, the charging particles or debris will be present. The charging process is the contact charging.

In addition to the contact charging and adsorption electrification, fracture electrification is another charging way. Specifically, the balance of positive and negative charge is broken when the solids fracture occurs. Therefore, the two separated parts carry an equal number of opposite charges. For example, the charging way is the fracture electrification when the material-loss of engine blades occurs. It can be clearly seen that the charge mode of particles in the aero-engine gas path is varied, and the charge characteristics relate to the physical, chemical, and other complex processes.

\section{The effect factors on the electrostatic charge in the aero-engine gas path}

The research results of the European PartEmis plan show that the carbon particle is about $6 \mathrm{~nm}$ measured in the aero gas turbine engine exhaust port. Studies by Arnold Sorokin show that there are two different particle diameter distributions in the engine exhaust, and the size of carbon 
particles is $5 \sim 7 \mathrm{~nm}$, and 20 30nm [12, 14]. During gas path component failure (such as blade rub) or combustion performance degradation, which results in the large-size carbon particles or metal abrasion, most of these abnormal particle sizes are larger than $40 \mu \mathrm{m}$ [15]. A large number of positive, negative ions and charged carbon particles are produced in the process of normal engine combustion. In addition, particles from the inlet collide with the random motion of the ion in a high temperature environment, and are charged. Charge quantities constitute the overall level of the electrostatic charge of the gas path. The charged process can be represented by the following formula [16]:

$$
q_{p}=\frac{d_{p} k T}{2 e^{2}} \ln \left(1+\frac{d_{p} v \pi e^{L} N t}{2 k t}\right)
$$

where $q_{p}$ is the charge of the particle ( $\left.p C\right)$, is the diameter of particle $(m)$, and $k$ is the Boltzmann constant $\left(1.38^{*} 10^{-23} \mathrm{~J} / \mathrm{K}\right)$, $T$ is the temperature $(K), e$ is electronic charge $\left(1.6^{*} 10^{-19}\right.$ $C), v$ is the mean of particles' velocity $(\mathrm{m} / \mathrm{s}), N$ is number concentration (ions $/ \mathrm{m}^{3}$ ), and $t$ is a time $(s)$. For aero-engines, the overall electrostatic charge of the gas path remains at a relatively stable level, and has a slight change with a change in work conditions. Once the work conditions of an aeroengine change, or the engine is faulty, a large number of abnormal particles will be the result. Such a fault could include component performance degradation in the engine gas path (combustor performance degradation) or failure (blades, rub-impact). Equation (6) indicates that the changes of temperature, particle concentration or particle size in the gas path can result in the changing of the overall electrostatic charge in the gas path. The real-time monitoring technology detects the change of the electrostatic charges in the engine gas path through the electrostatic sensor mounted at the exhaust pipe and provides early warning information about an engine fault.

Electrostatic sensors are developed for the real-time monitoring of the changes of the electrostatic charge in the gas path in the simulation experiments and engine bench experiments. The electrostatic sensor measurement model can be equivalent to [5]:

$$
U(t)=R_{C} q^{\prime}(t)
$$

Where $R_{C}$ is the equivalent resistance of the measurement circuit ( $M \Omega$ ), and $q$ is the induced charge on the electrostatic sensor probe $(p C)$. Therefore, the sensor output signal reflects the change of the charge on the sensor probe, and also reflects the changes in the sensitive space of the sensor. In other words, it detects the change of the electrostatic charge in the detection zone.

\section{Simulation experiment of electrostatic de- tection}

In this research, we build two experiment platforms to verify the electrostatic monitoring technology and the analysis results above. The first experiment platform we build is a simulation combustion environment. The experiment bench mainly contains of a fuel burner, and electrostatic sensor, and a data acquisition system (NIWLS-9234, a wireless dynamic signal acquisition produced by National Instruments). The electrostatic sensor is mounted at the outlet of the burner, and is used to detect changes in the electrostatic field, particularly in the sensing zone during the combustion process. The bench is used to verify the feasibility of real-time electrostatic monitoring, to simulate combustion performance degradation and combustion under conditions of various air-fuel ratios, and to simulate the metal particles present while a rub-fault of gas components occurs. The second experiment platform is a turbo-shaft engine test bench, which mainly includes the gas generator and turbine power output system. The gas generator consists of an annular inlet, a hybrid compressor (including a transonic axial compressor and a single-sided centrifugal compressor), combustion chamber, and twostage gas turbine, and the output power of the turbine is measured by the hydraulic dynamometer. The same sensor and data acquisition system are employed to detect the changes in the electrostatic field in the experiment. The sensor and system are mainly used to analyze factors that influence the change in the electrostatic field in the engine gas path. The schematic structure and configuration of the two test benches can refer to the literature listed in [6] and [10], respectively.

\subsection{The detection experiments of carbon particles}

According to the analysis above, the charge carried by the carbon particles is the primary part of the total electrostatic charge in the gas path. Therefore, the first experiment aims to detect the carbon particles and to verify the charge characteristics of carbon particles in the combustion process. The simulation experiments are performed on the simulation combustion bench. During the experiment, the combustor performance degradation, the fuel-rich combustion or lean combustion is simulated through setting the condition of the burner or adjusting the throttle of burners. For example, the carbon particles are generated when we decrease the intake air. Fig. 2 is the result of a combustion experiment. From Fig. 2, we can see that the output signal of the electrostatic 
sensor significantly changes at the time of ignition. The electrostatic charge level in the gas path obviously changes and the output voltage signal is negative. The induced charge on the sensor-sensing probe is reduced according to formula (7). The large carbon particle at the outlet of the burner has a positive charge. The analysis is consistent with the conclusion in the literature [13, 14].

\subsection{Experiment of detecting the metal particles}

Blade rub is one of the most common faults in the aeroengine gas path due to the work condition of the hightemperature, high-speed and high pressure in the aeroengine gas path. The larger size particles are produced at the time when the blasé rub fault occurs. The size of particles is usually greater than $40 \mu \mathrm{m}$. In this experiment, Fe particles

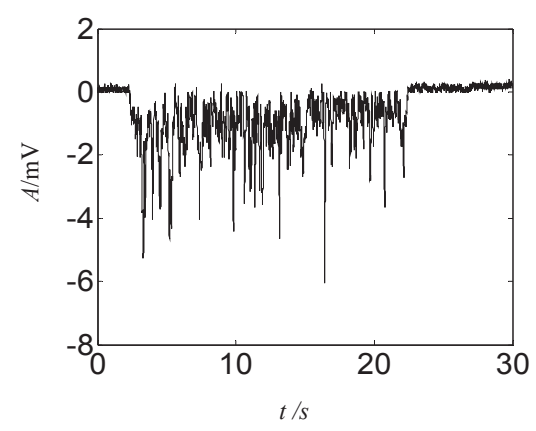

Fig. 2. The electrostatic signal induced by carbon particles

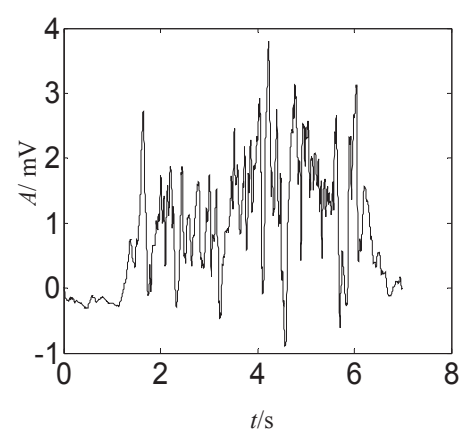

Fig. 3. The electrostatic signal induced by Fe particles

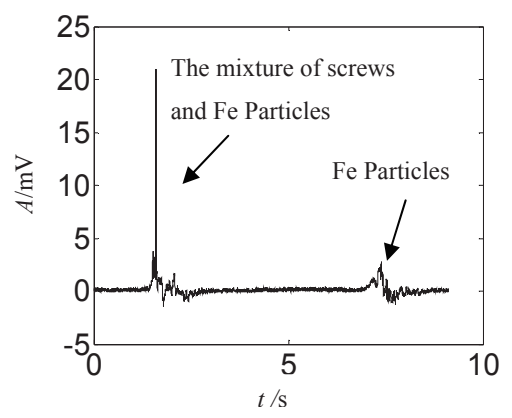

Fig. 4. The electrostatic signal induced by the mixture (particle size of approximately $75 \mu \mathrm{m}$ ) are injected to simulate the larger size metal abrasive particles produced due to the fault. The output signal of an electrostatic sensor in the experiment is shown in Fig. 3.

Furthermore, in order to verify the influence of particle size on the charge quantity in the experiment, the mixture of metal screws $(1 \mathrm{~mm})$ and iron powder are injected. The electrostatic monitoring signals are acquired during the experiment as shown in Fig. 4. As can be seen from the above two experiments, the polarities generated by the carbon particles and the metal particles are opposite, which can be used as the type identification of abnormal particle matter, and thus to distinguish the combustion system failure or mechanical failure. The larger the particle size, the greater the signal amplitude is induced. In Fig. 4, the first peak of the signal is generated by a mixed injection of metal screws and iron powder, and the second peak of the signal by an injection of iron powder only. The effect of larger particles on the electrostatic field of the experimental pipe is more obvious than the effect of smaller particles.

\subsection{The effect of the engine working condition on the electrostatic charge}

The gas path electrostatic monitoring experiment is performed on the turbo shaft engine test bench. The engine test process includes 14 stages; the output power of the engine and the duration of each stage are shown in Fig. 5. The electrostatic monitoring signal acquired in a complete aero-engine test process is shown in Fig. 6.

According to equation (7), the sensor output signal reflects the change of the induced charge on the sensor probe. The integral calculation of the output signal in the experiment is the quantity of the induced charge on the sensor probe, and the results are shown in Fig. 7. It illustrates the change trend of the electrostatic charge of the engine gas path in a complete test process.

As Fig. 5 and Fig. 7 show, the changes in the engine work condition (2->3, 6->7, 9->10, and 11-12) cause the changes in the electrostatic charge in the gas path, and the changes in the

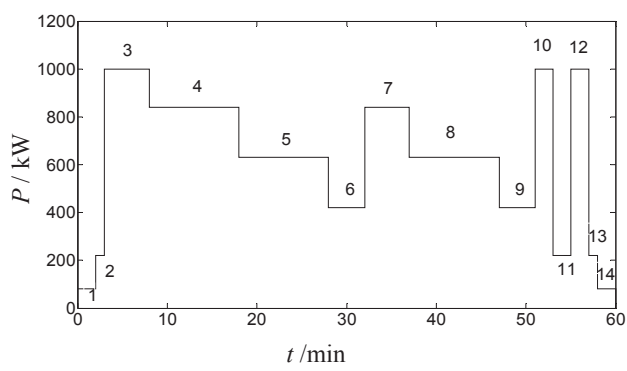

Fig. 5. The aero-engine test procedure 


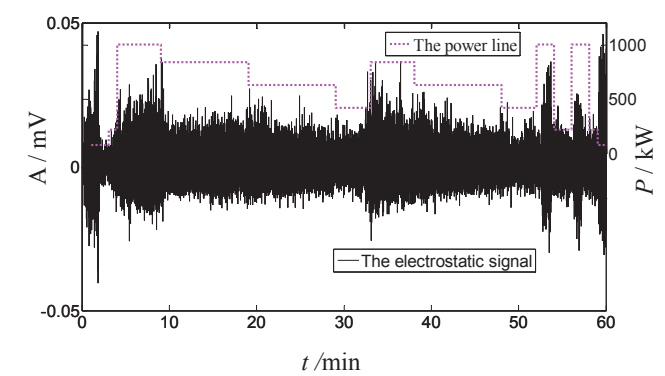

Fig. 6. The electrostatic monitoring signal in the engine test procedure

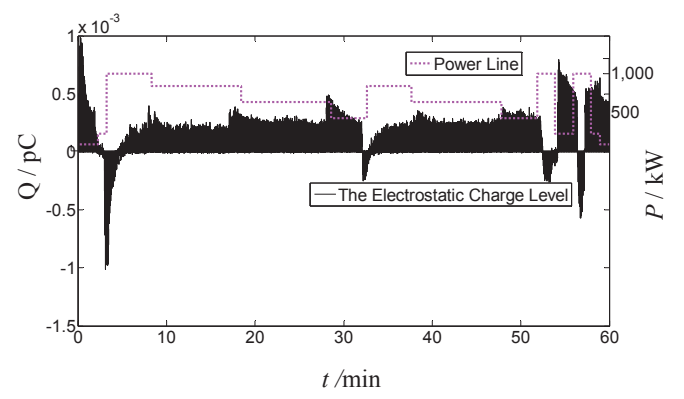

Fig. 7. The relationship between of the output power and the inductive charge

four stages are the engine power increases from little power to high power. This clearly shows that the rapid increase of the engine power will cause changes in the electrostatic charge in the gas path, and the induced charge on the sensor is negative in the moment when power increases and the induced charge on the sensor is positive in the relatively stable work condition. The major reasons for that can be briefly analyzed as follows. The incomplete combustion of the excess fuel injected when the power increase generates many larger carbon particles, and these carbon particles initially carry positive charge. Therefore, the induced charge on the sensor probe is negative. When the engine operates in stable work conditions, the main particles in the gas path are the small carbon particles carrying a negative charge, so the induced charge on the sensor probe is positive. The bench test demonstrates the capability of the electrostatic monitoring technology in the real-time monitoring of transient engine changes, but it also verifies that the large carbon particles have a positive charge initially, and the small carbon particles have a negative charge in the stable operating conditions. As metal particles can damage the engine, we do not conduct experiments that inject metal particles and that simulate the collision and friction fault on the engine test bench. How to implement the electrostatic monitoring technology on the engine test bench is the next concern.

\section{Conclusions}

1) The debris in the engine gas path includes particles produced by combustion and abnormal particles produced by the faults. The charge carried by carbon particles is the main part of the electrostatic charge in the aero-engine gas path.

2) There are many factors affecting the charge quantity of particles. Usually they are related to the particle formation process, the number of particles, and particle size. The large particle carries more charge quantities. The charge carried by carbon particles is negative, and the charge carried by metal particles is positive.

3) The engine test results show that the rapid increase of the engine power will change the electrostatic charge in the gas path, and the induced charge on the sensor is negative. While in a relatively stable operating condition, it is positive.

4) The electrostatic technology can effectively monitor the real-time transient change of the engine operating conditions.

\section{Acknowledgments:}

The work was supported by the National Natural Science Foundation of China (51105344). It was also partly supported by the Aeronautical Science Foundation of China(2012ZB55003, 2013ZB55003), the Basic Research and Advanced Technology Program of Henan Province, China (132300410269), the key project of Henan educational department, China (14A590001) and the foundation of Henan educational department for the young core teachers in university (2014GGJS-104). The authors also wish to thank these organizations for their financial support.

\section{References}

[1] Carter T J., "Common failures in gas turbine blades", Engineering Failure Analysis, Vol. 12, No. 2, 2005, pp. 237-247.

[2] Balthasar M, Mauss F, and Wang H., "A computational study of the thermal ionization of soot particles and its effect on their growth in laminar premixed flames", Combustion and Flame, Vol. 129, No. 1-2, 2002, pp. 204-216.

[3] Robert P. Couch., "Detecting Abnormal Turbine Engine Deterioration Using Electrostatic Methods", Journal of Aircraft, Vol. 15, No. 10, 1978, pp. 692-695.

[4] Novis A, Powrie H, and Novis A., "PHM Sensor Implementation in the Real World - a Status Report", IEEE Aerospace Conference Proceedings, 2006, pp. 1-9. 
[5] Wen Zhenhua, Zuo Hongfu, Pecht, M.G., "Electrostatic monitoring of gas path debris for aero-engines", IEEE Transactions on Reliability, Vol. 60, No. 1, 2011, pp. 33-40.

[6] Wen Zhenhua, Zuo Hongfu, and Li Yaohua., "Gas path debris electrostatic monitoring technology and experiment", Journal of Aerospace Power, Vol. 23, No. 12, 2008, pp. 23212326.

[7] Wen Zhenhua, Zuo Hongfu, and Wang Hua, et al., "Characters of sensor for aero engine gas path electrostatic monitoring", Transducer and Micro-system Technologies, Vol. 27, No. 11, 2008, pp. 28-31.

[8] Wen Zhenhua, and Zuo Hongfu., "Electrostatic Monitoring Signal Processing Method for Aero-engines Gas Path Based on ICA", Mechanical Science and Technology for Aerospace Engineering, Vol. 30, No. 11, 2011, pp. 1872-1876.

[9] Wen Zhenhua, Zuo Hongfu, and Li Yaohua., "New method for aero engine gas path monitoring", Journal of Nanjing University of Aeronautics \& Astronautics, Vol. 41, No. 2, 2009, pp. 248-252.

[10] Li Yaohua, Zuo Hongfu, and Liu Pengpeng., "Gas path electrostatic monitoring of turbo-shaft engine: an exploratory experiment", Acta Aeronautica et Astronautica
Sinica, Vol. 31, No. 11, 2012, pp. 2174-2181.

[11] Yan Chuanjun, and Fan Wei., Combustion theory, Northwestern Polytechnical University Press, Xi'an, 2005.

[12] Sorokin A, Vancassel X, and Mirabel P., "Emission of ions and charged soot particles by aircraft engines", "Atmospheric Chemistry and Physics", Vol. 3, No. 2, 2003, pp. 325-334.

[13] Sorokin, A, and Arnold, F., "Organic positive ions in aircraft gas-turbine engine exhaust", Atmospheric Environment, Vol. 40, No. 32, 2006, pp. 6077-6087.

[14] Sorokin, A, and Arnold, F., "Electrically charged small soot particles in the exhaust of an aircraft gas-turbine engine combustor: comparison of model and experiment", Atmospheric Environment, Vol. 37, No. 17, 2004, pp. 26112618.

[15] Powrie H, and Novis A., "Gas path debris monitoring for F-35 joint strike fighter propulsion system PHM", Proceeding of Proceedings of IEEE Aerospace Conference. Montana, USA, 2006, pp. 1-8.

[16] Ren Jun, Shen Jian, and Lu Shouci, Particle dispersion Science and Technology, Chemical Industry Press, Beijing, 2005, pp. 124-129. 